\title{
Pulmonary function and respiratory symptoms in a population of airport workers
}

\author{
W S Tunnicliffe, S P O’Hickey, T J Fletcher, J F Miles, P S Burge, J G Ayres
}

\begin{abstract}
Objectives-To assess the prevalence of respiratory symptoms and to measure spirometry in a sample of employees of Birmingham International Airport, United Kingdom, to examine whether occupational exposure to aircraft fuel or jet stream exhaust might be associated with respiratory symptoms or abnormalities of lung function.
\end{abstract}

Methods-Cross sectional survey by questionnaire and on site measurement of lung function, skin prick tests, and exhaled carbon monoxide concentrations. Occupational exposure was assigned by job title, between group comparison were made by logistic regression analysis.

Results-222/680 full time employees were studied (mean age 38.6 y, $63 \%$ male, $28 \%$ current smokers, $6 \%$ self reported asthma, $19 \%$ self reported hay fever). Upper and lower respiratory tract symptoms were common and $51 \%$ had one or more positive skin tests. There were no significant differences in lung function tests between exposure groups. Between group comparisons of respiratory symptoms were restricted to male members of the medium and high exposure groups. The adjusted odds ratio (OR) for cough with phlegm and runny nose were found to be significantly associated with high exposure (OR 3.5, 95\% confidence interval (95\% CI) 1.23 to 9.74 and $2.9,1.32$ to 6.40 respectively) when the measured confounding effects of age and smoking, and in the case of runny nose, self reported hay fever had been taken into account. There was no obvious association between high exposure and the presence of shortness of breath or wheeze, or for the symptoms of watering eyes or stuffy nose.

Conclusions-These findings support an association in male airport workers, between high occupational exposures to aviation fuel or jet stream exhaust and excess upper and lower respiratory tract symptoms, in keeping with a respiratory irritant. It is more likely that these effects reflect exposure to exhaust rather than fuel, although the effects of an unmeasured agent cannot be discounted.

(Occup Environ Med 1999;56:118-123)

Keywords: respiratory symptoms; airport workers; occupational exposure; aviation fuel; jet stream exhaust

Concern about the possible adverse effects of environmental pollution is now widespread and much of this concern has focused on the role of atmospheric pollutants in respiratory disease. ${ }^{1-4}$ There is now growing evidence for adverse respiratory health effects from exposures to among others, oxides of nitrogen, ${ }^{5}$ ozone,${ }^{6}$ sulphur dioxide, ${ }^{7}$ and particulates ${ }^{8}$ either singularly or in combination - such as in motor vehicle exhaust fumes. ${ }^{9} 10$

It was suggested to us by patients with asthma living near Birmingham International Airport that exposure to aviation fuel or jet stream might exacerbate respiratory symptoms. This possibility is supported by findings linking domestic exposure to kerosene heaters, ${ }^{11}$ and ambient volatile organic compounds, ${ }^{12}$ with respiratory symptoms. We hypothesised that exposure to aviation fuels or jet stream might be associated with respiratory symptoms and selected the workforce of Birmingham International Airport as a potentially suitable population in which to test this hypothesis.

Occupational exposures to aviation fuel and jet stream exhausts usually occur independently and if both agents are capable of producing respiratory morbidity, the patterns of symptoms caused by each might be expected to differ. Jet exhaust emissions are very similar to emissions from standard internal combustion engines except that they do not contain oxides of sulphur or lead. The efficiency of jet engines is reduced during taxiing and combustion is incomplete, resulting in higher emissions of unburned hydrocarbons. Despite this, $<0.1 \%$ of hydrocarbons escape unburned even when taxiing (P Walker, personal communication, Rolls Royce, Derby).

Significant exposure of personnel to aviation fuel is likely to occur only accidentally and during refuelling operations. Transient plumes of fuel aerosols may also be generated at the time of engine ignition. As a consequence fuel exposure would usually involve only a few personnel and then only intermittently. Review of safety data ${ }^{13}{ }^{14}$ and clinical research examining the effects of exposure to aviation fuel and other volatile hydrocarbon $\mathrm{s}^{15}{ }^{16}$ suggest that any effects of such exposure might be expected to be highly time dependent.

\section{Methods}

STUDY DESIGN

The study was cross sectional in design. Data were gathered through a self administered questionnaire and lung function and skin tests were performed on site at a later date.

\section{STUDY POPULATION}

The airport is in the south east of the West Midlands conurbation in the predominantly 
residential borough of Solihull. Most airport employees live locally and commute to work daily. All members of airport staff, excluding contract workers and airline employees (pilots, air stewards and stewardesses, check in staff) were identified by the personnel department and informed of the study.

\section{EXPOSURE CLASSIFICATION AND RANDOM} SAMPLING

On the basis of official job titles, all full time members of the work force were classified by one of the investigators (SP O'H) before the morbidity survey, into three exposure groups depending on their likely exposure to aviation fuel or jet stream. The high exposure group consisted of baggage handlers, airport hands, marshallers, operational engineers, fitters, and engineering technicians. All would expect to spend a considerable proportion of their working day in close proximity to in service aircraft. The medium exposure group consisted of among others, security staff, fire fighters, and airfield operations managers, who would expect to spend some of their working time on the airport apron, some in reasonable proximity to aircraft, and some within the terminal buildings. The low exposure group consisted of terminal and office workers.

Random sampling was performed and those identified were sent a questionnaire and asked to visit a room in the main terminal building, during their usual working shift, on any one of five study days for measurement of lung function and skin prick testing.

QUESTIONNAIRE

The self administered 76 point questionnaire (available on request) was sent to subjects through the airport's internal mail system. Demographic and employment details were requested as well as information relating to respiratory health, smoking, and family history. Individual questions were taken from previously validated questionnaires-such as the MRC respiratory questionnaire and the IUATLD bronchial symptom questionnaire ${ }^{17}$-along with a series of study specific questions about work patterns.

PULMONARY FUNCTION

Subjects' dynamic lung volumes were measured with one of two dry bellow spirometers or by a pneumotachograph (Vitalograph, Bucks, UK). Technicians were blinded to exposure and the instrument used was randomly assigned. The best of three reproducible and technically acceptable blows was used for analysis. European Community for coal and steel predicted values were used. ${ }^{18}$

SKIN PRICK TESTING

Skin prick tests were performed with positive (histamine) and negative control solutions and Dermatophagoides pteronyssinus, cat, grass, mixed tree, and Aspergillus fumigatus allergen solutions (Pharmacia, Sweden). The mean weal diameters ${ }^{19}$ were read after 15 minutes. We defined a positive response to any allergen as a mean weal diameter for that allergen larger by $\geqslant 3 \mathrm{~mm}$ than that of the negative control solution.

EXHALED CARBON MONOXIDE MEASUREMENTS A hand held micro smokerlyzer (Bedfont Scientific, England) was used to measure exhaled carbon monoxide concentrations. Subjects were asked to inhale and then hold their breath for 15 seconds, before exhaling slowly into the meter.

\section{ANALYSIS}

Data from the completed questionnaires, lung function measurements, carbon monoxide readings, and skin test results were entered into a personal computer. Analyses were performed with Epi-Info version 5 (USD, USA) and Stata release 3.1 (Stata, USA).

The results of the pulmonary function tests and the responses to each of the following questions were used as the main outcome measures in the analysis.

- Do you cough ?

- Do you cough up phlegm from your chest?

- In the past 12 months have you had irritation or watering of the eyes?

- In the past 12 months have you had more than two episodes of a stuffy nose ?

- In the past 12 months have you had more than two episodes of a runny nose ?

- In the past 12 months have you had wheezing or whistling in your chest?

- On your worst day in the last 12 months, were you troubled by shortness of breath when hurrying on level ground or walking up a slight hill ?

Respondents were also asked to record if their symptoms improved on days away from work.

Prevalences for each of the symptoms were calculated across the exposure categories, as were the means of the percentage predicted forced expiratory volume in one second $\left(\mathrm{FEV}_{1}\right)$, forced vital capacity (FVC) measurements, and the exhaled carbon monoxide results. Lung function and the mean exhaled carbon monoxide values were compared by analysis of variance (ANOVA), and the proportions of subjects with positive skin prick test results were compared by $\chi^{2}$ test. Multiple logistic regression was used to evaluate the association between exposure group and symptoms, while adjusting for age, smoking, and where appropriate, a self reported history of asthma or hay fever. The data did not contain a variable that independently reflected socioeconomic status and a quantitative assessment of past smoking could not be derived from the recorded smoking related variables.

The study protocol was approved by the Birmingham Heartlands Hospital ethics committee, subject to all participants providing written consent before entering the study. The approval of the airport management, safety committee, and unions was also obtained. 


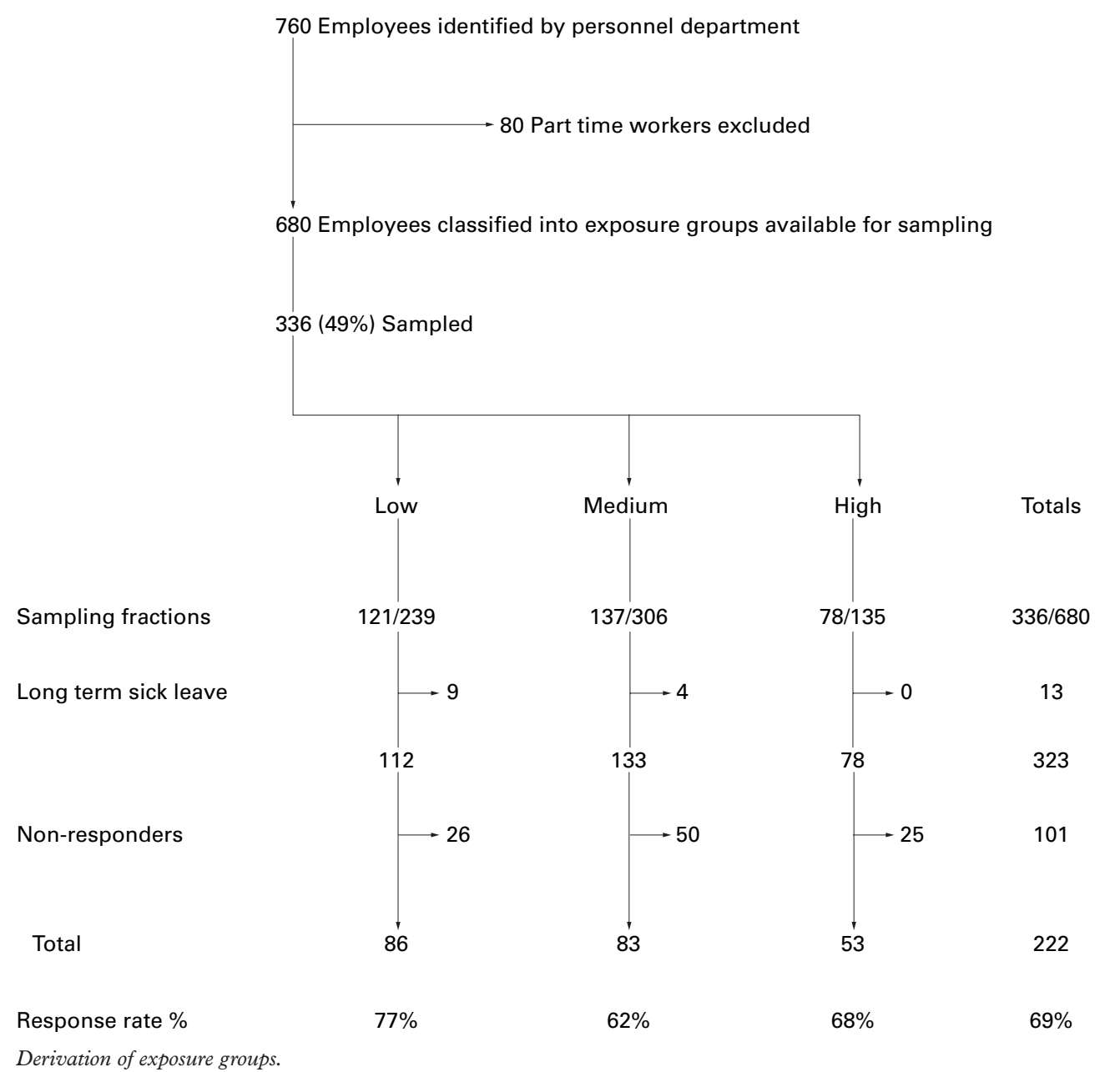

\section{Results}

STUDY POPULATION AND RESPONSE RATE

Figure 1 shows the study population and response rate. Six hundred and eighty full time employees were identified by the airport's personnel department. On the basis of job title, 239 were categorised as the low exposure group, 306 as medium, and 135 as high. Random sampling resulted in 336 potential participants, 121 from the low, 137 from the medium, and 78 from the high exposure group. In total, 222 of the 336 randomly sampled employees returned questionnaires and completed the study.

GROUP CHARACTERISTICS AND DEMOGRAPHY

Table 1 shows the demographic characteristics of participants, duration of employment at the airport, and the median time per day spent in the aircraft taxiing area. Members of the high exposure group spent most of their working day in the aircraft taxiing area whereas members of the low exposure group spent almost none of their working day there; the median duration for those in the medium exposure group was 1 hour, validating the initial exposure classification. Comparisons across exposure groups showed similarity for age, duration of employment, and atopy, but not for the other characteristics. The differences in sex distribution between the groups were considerable and reported smoking also

varied between groups. Exhaled carbon monoxide results are listed by exposure group, stratified by smoking; among the ex-smokers and never smokers, the mean exhaled carbon monoxide concentrations rose with increasing exposure but these differences were not significant. The proportion of subjects who reported that they had asthma was small in each group but the prevalence of self reported hay fever in the low exposure group was around twice that of the medium and high exposure groups; $28 \%$ $v 14 \%$ and $13 \%$ respectively $(\mathrm{p}=0.037)$.

\section{LUNG FUNCTION}

Table 2 shows the lung function results by exposure group, stratified by smoking. There were no significant differences in lung function within or between the exposure groups.

CRUDE PREVALENCE OF RESPIRATORY SYMPTOMS Table 3 lists the crude prevalences, by exposure group for the seven symptoms used as the main outcome measures. We had hoped to be able to compare these crude rates across the exposure groups, but the differences in the group characteristics suggested that any such comparisons should be treated with caution.

CRUDE AND ADJUSTED ODDS RATIOS

To enable more valid between group comparisons we adopted a strategy of restriction followed by the use of multiple logistic 
Table 1 Characteristics of the airport workers studied by exposure category

\begin{tabular}{|c|c|c|c|c|}
\hline & All & Low & Medium & High \\
\hline $\mathrm{n}$ & 222 & 86 & 83 & 53 \\
\hline Age (y, mean (SD)) & $38.6(10.4)$ & $38.7(11.2)$ & $39.8(10.0)$ & $36.7(8.3)$ \\
\hline \multicolumn{5}{|l|}{ Sex: } \\
\hline Men $(\mathrm{n}(\%))$ & (63) & (37) & $(66)$ & $53(100)$ \\
\hline Women (n) & 82 & 54 & 28 & 0 \\
\hline Duration of employment (y, mean (SD)) & $7.6(6.3)$ & $6.7(5.8)$ & $8.1(6.1)$ & $8.4(6.8)$ \\
\hline \multicolumn{5}{|l|}{ Smoking: } \\
\hline Never (n (\%)) & $103 \quad(46)$ & $52(60)$ & $28 \quad(34)$ & $23 \quad(43)$ \\
\hline Exhaled CO (mean (SD), ppm) ${ }^{\star}$ & $3.8(2.3)$ & $3.2(2.2)$ & $4.0(2.3)$ & $5.1(2.3)$ \\
\hline Ex-smoker (n (\%)) & $56 \quad(26)$ & $19 \quad(23)$ & $25 \quad(30)$ & 12 (23) \\
\hline Exhaled CO (mean (SD), ppm)* & $3.5(2.3)$ & $3.2(2.3)$ & $3.4(2.4)$ & $4.3(2.5)$ \\
\hline Current (n (\%)) & $63(28)$ & $15 \quad(17)$ & $30 \quad(36)$ & $18 \quad(34)$ \\
\hline Exhaled CO (mean (SD), ppm) ${ }^{\star}$ & $27.2(16.8)$ & $27.2(18.3)$ & $27.4(17.9$ & $27.1(14.6)$ \\
\hline Self reported asthma (n (\%)) & $13(6)$ & $5(6)$ & $3(4)$ & 5 (9) \\
\hline Self reported hayfever (n (\%)) & $43 \quad(19)$ & $24 \quad(28)$ & (14) & (13) \\
\hline Atopy on skin tests $(\mathrm{n}(\%)) \dagger$ & $111 / 216$ & $40 / 83$ & $42 / 81$ & $29 / 52$ \\
\hline \multicolumn{5}{|l|}{ Time in aircraft taxiing area } \\
\hline (h/day, median (interquartile range)) & - & $(0-1)$ & $(0-6)$ & $(4-8)$ \\
\hline
\end{tabular}

ॠExhaled CO concentrations (parts per million).

†Atopy $=\geqslant 1$ positive skin tests to allergen. Missing values, denominators shown.

regression analysis. The presence of an all male high exposure group precluded the initial use of logistic regression to control for the confounding effects of sex and consequently we elected to restrict our analyses to only male respondents. Also, and as a consequence of the use of job title to classify exposure, we anticipated that socioeconomic factors might completely confound the exposure of the male members of one or more of our groups. This was found to be highly probable as the male members of the low exposure group were all found to be members of the airport management team, whereas the medium and high exposure groups both comprised manual, skilled, and highly skilled employees. On this basis, we decided to also restrict our analyses to comparisons between only the medium and high exposure groups. We then used multiple logistic regression to evaluate the association in these men between high exposure and the chosen symptoms, correcting for age and smoking, and where appropriate, for the presence of self reported hay fever or asthma.

Table 2 Lung function measurements in each exposure group stratified by smoking

\begin{tabular}{llrrr}
\hline Smoking & Variable & Low & Medium & \multicolumn{1}{c}{ High } \\
\hline Never smoked & $\mathrm{n}$ & 52 & 28 & 23 \\
& $\mathrm{FEV}_{1}$ & $101(12)$ & $102(12)$ & $100(12)$ \\
& $\mathrm{FVC}$ & $107(14)$ & $108(12)$ & $106(11)$ \\
Ex-smokers & FEV $_{1} /$ FVC & $81(14)$ & $78(16)$ & $78(16)$ \\
& $\mathrm{n}$ & 19 & 25 & 12 \\
& $\mathrm{FEV}_{1}$ & $97(15)$ & $102(15)$ & $99(11)$ \\
& $\mathrm{FVC}$ & $107(12)$ & $109(17)$ & $106(11)$ \\
Current smokers & $\mathrm{FEV} / \mathrm{FVC}$ & $77(15)$ & $78(11)$ & $77(10)$ \\
& $\mathrm{n}$ & 15 & $30(12)$ & 18 \\
& $\mathrm{FEV}_{1}$ & $94(16)$ & $100(12)$ & $93(11)$ \\
& $\mathrm{FVC}$ & $108(18)$ & $109(12)$ & $104(11)$ \\
& $\mathrm{FEV}_{1} /$ FVC & $76(15)$ & $77(9)$ & $74(12)$
\end{tabular}

$\mathrm{FEV}_{1} \mathrm{~s}$ and FVCs expressed as means (SD) of \% predicted value achieved.

There were no significant differences in mean lung function values within or between exposure groups.

Table 3 Crude prevalence ( $(\%)$ ) of reported symptoms by exposure category

\begin{tabular}{lrrrr}
\hline & \multicolumn{1}{c}{ All (22) } & Low (86) & Medium (83) & High (53) \\
\hline Cough & $99(45)$ & $37(43)$ & $30(36)$ & $32(60)$ \\
Cough with phlegm & $50(23)$ & $18(21)$ & $13(16)$ & $19(36)$ \\
Watering eyes & $86(39)$ & $39(45)$ & $31(37)$ & $16(30)$ \\
Stuffy nose & $123(55)$ & $43(50)$ & $46(55)$ & $34(64)$ \\
Runny nose & $105(47)$ & $39(45)$ & $35(42)$ & $31(58)$ \\
Wheeze or whistling & $52(23)$ & $21(24)$ & $18(22)$ & $13(25)$ \\
Shortness of breath` & $38 / 218(17)$ & $17 / 84(20)$ & $14 / 82(17)$ & $7 / 52(13)$ \\
\hline
\end{tabular}

${ }^{\star}$ Missing values, denominators shown.
Table 4 shows the crude and adjusted odds ratios (ORs) for the male members of the high exposure group relative to the male members of the medium exposure group, for each of the symptoms.

The adjusted ORs for cough with phlegm, and runny nose were significantly associated with high exposure (ORs 3.5 (95\% confidence interval (95\% CI) 1.23 to 9.74$)$ and $2.9(95 \%$ CI 1.32 to 6.40 ) respectively) when the measured confounding effects of age, smoking, and in the case of runny nose, self reported hay fever had been taken into account. The adjusted OR for cough remained increased (2.2) but not significantly so. Of note, there was no obvious association between high exposure and the presence of shortness of breath or wheeze, or for the symptoms of watering eyes or stuffy nose.

We also attempted to perform subgroup analysis of those reporting symptoms. We examined the prevalence of improved symptoms when away from work by exposure group. Unfortunately, the absolute numbers in each

Table 4 Crude and adjusted ORs (95\% CIs) for symptoms in men in the high exposure group

\begin{tabular}{|c|c|c|c|}
\hline \multirow[b]{2}{*}{ Symptom } & \multirow{2}{*}{$\begin{array}{l}\text { Medium } \\
\text { OR }\end{array}$} & \multicolumn{2}{|l|}{ High } \\
\hline & & $O R$ & $95 \% C I$ \\
\hline \multicolumn{4}{|l|}{ Cough: } \\
\hline Crude & 1.0 & $2.3^{\star}$ & 1.06 to $4.94 \dagger$ \\
\hline Adjusted + & 1.0 & 2.2 & 0.94 to 5.26 \\
\hline \multicolumn{4}{|c|}{ Cough with phlegm: } \\
\hline Crude & 1.0 & $3.3^{\star}$ & 1.29 to $8.83 \dagger$ \\
\hline Adjusted $\dagger$ & 1.0 & $3.5^{\star}$ & 1.23 to $9.74 \dagger$ \\
\hline \multicolumn{4}{|l|}{ Watering eyes: } \\
\hline Crude & 1.0 & 0.7 & 0.31 to 1.60 \\
\hline Adjusted $\ddagger$ & 1.0 & 0.8 & 0.32 to 1.86 \\
\hline \multicolumn{4}{|l|}{ Stuffy nose: } \\
\hline Crude & 1.0 & 1.6 & 0.71 to 2.90 \\
\hline Adjusted $\ddagger$ & 1.0 & 1.4 & 0.49 to 2.81 \\
\hline \multicolumn{4}{|l|}{ Runny nose: } \\
\hline Crude & 1.0 & $3.2^{\star}$ & 1.38 to $6.77^{\star}$ \\
\hline Adjusted $\ddagger$ & 1.0 & $2.9^{\star}$ & 1.32 to $6.40^{\star}$ \\
\hline \multicolumn{4}{|c|}{ Wheeze or whistling in chest: } \\
\hline Crude & 1.0 & 1.4 & 0.36 to 3.02 \\
\hline Adjusted $₫$ & 1.0 & 0.8 & 0.31 to 2.73 \\
\hline \multicolumn{4}{|c|}{ Shortness of breath: } \\
\hline Crude & 1.0 & 0.8 & 0.31 to 2.01 \\
\hline Adjusted $\dagger$ & 1.0 & 0.9 & 0.34 to 2.52 \\
\hline
\end{tabular}

${ }^{\star} \mathrm{p}<0.05$.

tAdjusted for age and smoking.

$\ddagger$ Adjusted for age, smoking and self reported history of hayfever. \Adjusted for age, smoking and self reported history of asthma. 
subgroup was small (data not shown) and we were unable to show an association between exposure and any improved symptoms when away from work.

\section{Discussion}

The aim of this study was to examine whether occupational exposure to aviation fuel or jet stream exhaust might be associated with respiratory symptoms in a population of airport workers. Due to the differences in the demographic make up of the exposure groups we thought it wise only to make comparisons between outcomes recorded in male members of the medium and high exposure groups. Allowing for measured confounding through the use of logistic regression analysis, the outcomes cough with phlegm and runny nose (3.5 and 2.9 respectively) were found to be significantly associated with high exposure; whereas the OR for cough in the high exposure group (2.2) was increased, this was nonsignificant.

In those with symptoms, we were unable to show an association between exposure and improvement in symptoms when away from work. This was not wholly unexpected as subgroup analysis resulted in small absolute numbers and a type 2 error may have prevented us from detecting such an association even if one was truly present; also the population mostly lived close to the airport and might be exposed to jet stream on days away from work. There was no obvious association between high exposure and the symptoms of wheeze or shortness of breath, nor for watering eyes or stuffy nose. Also, no differences in lung function between groups were detected.

Overall these results support the hypothesis that occupational exposure to jet stream exhaust or jet fuel is associated with an excess of some respiratory symptoms in male airport workers. The pattern of respiratory symptoms is compatible with exposure to a respiratory irritant, producing, in part an element of occupational bronchitis. This study yields no evidence in support of an association between such exposure and an excess of breathlessness or wheeze, suggestive of occupational asthma.

Before accepting these findings, several fundamental constraints and the possible effects of bias and residual confounding need to be carefully considered:

\section{CONSTRAINTS}

The cross sectional nature of the study limited outcome variables to the period prevalence of persistent, respiratory symptoms and once only measures of respiratory function. We must accept that any acute or severe illnesses, or transient alteration in lung function potentially associated with exposure, if present, would have been likely to have been missed. The demographic characteristics and size of the population samples under study also limited the application and power of standard statistical techniques.

Also, the collection of details about exposures to the suspect agents (dose rate, accumulated dose, dose peaks, and frequency of expo- sures) and recognised confounders was also limited. A clear definition of what constitutes exposure is an essential component of any epidemiological hypothesis being tested in the occupational field. The usefulness of such a study depends to a large extent on the amount, specificity, and precision of the exposure data recorded. Despite this limitation, the recorded concentrations of exhaled $\mathrm{CO}$ and the average number of hours a day each group spent in the aircraft taxiing area offered support for our exposure classification.

BIAS AND RESIDUAL CONFOUNDING

The response rates of the medium and high exposure groups were similar (62\% and $68 \%)$ and this is unlikely to explain the observed differences. Recall bias, however, cannot be as easily discounted. The medium exposure group included the airport firemen; due to the nature of their job, firemen may be less likely to report respiratory symptoms, and on the whole, might be expected to be physically fitter than most other groups of employees. The differences we found may in fact represent, in part, a healthy worker effect although the outcome runny nose is difficult to explain on this basis.

Residual confounding due to smoking also needs to be considered. Although current smoking rates were similar in the medium and high exposure groups, a positive association between exposure and accumulated pack-years or current number of cigarettes smoked a day cannot be excluded. Although no difference was found in mean exhaled CO concentrations between the smokers in these groups, this should not be interpreted as evidence against such an association.

Confounding due to social class also needs to be considered. No individual measure of socioeconomic status was recorded for the study participants, but the job categories of workers classified in both the medium and high exposure groups comprised unskilled, skilled, and highly skilled occupations. Although confounding by this characteristic cannot be excluded, it seems an unlikely explanation for the observed differences.

Spirometry did not detect any significant differences in lung function between exposure groups or between current smokers, exsmokers, and never smokers. The absence of detectable abnormalities in current smokers and ex-smokers may reflect the relatively young age of the work force studied or possibly a healthy smoker effect.

\section{Conclusion}

The increased adjusted ORs for cough with phlegm and runny nose in members of the high exposure group are likely to reflect a true association between these symptoms and their occupational setting, although bias and residual confounding may be in part responsible. The nature of these symptoms are compatible with exposure to a respiratory irritant and while a fuel or fume effect cannot be discounted, we think that this is more likely to reflect a jet stream exhaust effect. 
We gratefully acknowledge the cooperation of the participants in this study and the excellent technical and research assistance of Cindy Billingham, Sue Cattell, Linda Webber, and the staff of Birmingham International Airport's occupational health department. The study was supported by the Heartlands Research Institute, Birmingham Heartlands Hospital. No direct or indirect funding was provided by any other organisation for this study.

1 Anonymous. Environmental pollution: it kills trees, but does it kill people? Lancet 1992;340:821-2.

2 Burnett RT, Dales R, Krewski D, et al. Associations between ambient particulate sulphate and admissions to Ontario hospitals for cardiac and respiratory diseases. $A m \mathcal{F}$ Epidemiol 1995;142:15-22.

3 Ostro BD, Lipsett MJ, Mann JK, et al. Air pollution and respiratory morbidity among adults in southern California. Am ₹ Epidemiol 1993;137:691-700.

4 Sunyer J, Saez M, Murillo C, et al. Air pollution and emergency room admissions for chronic obstructive pulmonary disease: a five year study. Am F Epidemiol 1993;137:701-5.

5 Hasselblad V, Kotchman DJ, Eddy DM. Synthesis of environmental evidence: nitrogen dioxide epidemiology studies. F Air Waste Manag Assoc 1992;42:662-71.

6 Studies. F Air Waste Manag Assoc 1992;42:662-71. Spektor DM, Thurston GD, Mao J, et al. Effects of singleand multi-day ozone exposures on respiratory function

7 Linn WS, Avol EL, Peng RC, et al. Replicated dose-response study of sulphur dioxide in normal, atopic, and asthmatic volunteers. Am Rev Respir Dis 1987;136:1127-34.

8 Dockery DW, Pope III CA. Acute respiratory effects of particulate air pollution. Annu Rev Public Health 1994;15:10732.

9 Clench-Aas J, Larssen S, Bartonova A, et al. The health effects Lillestrom: Norwegian Institute for Air Research, 1991.
10 Edwards J, Walters S, Griffiths RK. Hospital admissions for asthma in pre-school children: relationship to major roads in Birmingham,

11 Cooper KR, Alberti RR. Effect of kerosene heater emissions on indoor air quality and pulmonary function. Am Rev Respir Dis 1984;129:629-31.

12 Ware JH, Spengler JD, Neas LM, et al. Respiratory and irritant health effects of ambient volatile organic compounds. The Kanawha County health study. Am 7 Epidemiol 1993; 137:1287-301.

13 Shell UK product safety data sheet No V10001 version 13 05 88. Shell aviation gasolines. 100LL. Shell-Mex House, Strand, London: Shell UK.

14 Shell UK Product safety data sheet No V20001 version 13 05 88. Shell aviation turbine fuels (kerosines). Jet A-1, Shell Jet A-1 with AL38. Shell-Mex House, Strand, London: Shell UK.

15 Harving H, Dahl R, Mohave L. Lung function and bronchial reactivity in asthmatics during exposure to volatile organic compounds. Am Rev Respir Dis 1991;143:751-4.

16 Pfaff J, Parton K, Lantz R, et al. Effects of JP-8 jet fuel exposure on pulmonary function Am Rev Respir Dis 1992; 145(suppl):A89.

17 Burney PGJ, Laitinen LA, Perdizet S, et al. Validity and repeatability of the IUATLD (1984) bronchial symptoms questionnaire: an international comparison. Eur Respir $\mathcal{F}$ 1989;2:940-5.

18 Quanjer $\mathrm{PhH}$, ed. Standardised lung function testing. Report to the Working Party for Standardisation of Lung Function Tests, European Community for Coal and Steel. Bull Europ Physiopath Respir 1983;19(suppl 5):1-95.

19 Bernstein IL. Proceedings of the taskforce on guidelines for standardising old and new technologies used for the diagnosis and treatment of allergic disease. 7 Allergy Clin Immunol 1988;82:487-505.

\section{Rejected manuscripts}

From February 1994, authors whose submitted articles are rejected will be advised of the decision and one copy of the article, together with any reviewer's comments, will be returned to them. The fournal will destroy remaining copies of the article but correspondence and reviewers' comments will be kept. 